\title{
LncRNA Testis-specific transcript, Y-linked 15 (TTTY15) promotes proliferation, migration and invasion of colorectal cancer cells via regulating miR-29a-3p/DVL3 axis
}

\section{Ming-zheng Cao}

Linyi Central Hospital

\section{Ying Ba}

Linyi Central Hospital

Yue-feng Li ( $\nabla$ xuancilen703@163.com )

Linyi Central Hospital https://orcid.org/0000-0001-6807-3730

\section{Primary research}

Keywords: LncRNA TTTY15, miR-29a-3p, DVL3, Colorectal cancer

Posted Date: March 10th, 2020

DOI: https://doi.org/10.21203/rs.3.rs-16624/v1

License: (c) (i) This work is licensed under a Creative Commons Attribution 4.0 International License.

Read Full License 


\section{Abstract}

Background: Long non-coding RNA TTTY15 is oncogenic in prostate cancer, however its expression and function in colorectal cancer remain largely unknown.

Methods: Paired colorectal cancer samples/adjacent tissues were collected, and the expression levels of TTTY15, miR-29a-3p and disheveled segment polarity protein 3 (DVL3) were examined by quantitative real-time polymerase chain reaction (qRT-PCR). TTTY15 shRNAs were transfected into HT-29 and HCT116 cell lines using lipofectamine reagent; the proliferation and colony formation were detected by CCK-8 assay and plate colony formation assay. qRT-PCR and western blot were used to analyze the changes of miR-29a-3p and DVL3; luciferase reporter gene assay was used to determine the regulatory relationship between miR-29a-3p and TTTY15, miR-29a-3p and DVL3.

Results: TTTY15 was significantly up-regulated in cancerous tissues of colorectal cancer samples, positively correlated with the expression of DVL3, while negatively correlated with miR-29a-3p. After TTTY15 shRNAs were transfected into colorectal cancer cells, the proliferation and metastasis of cancer cells were significantly inhibited. TTTY15 shRNAs could reduce the expression of DVL3 on both mRNA and protein levels, and the luciferase activity of TTTY15 sequence was also inhibited by miR-29a-3p. DVL3 was also validated as a target gene of miR-29a-3p.

Conclusion: TTTY15 is abnormally upregulated in colorectal cancer tissues, and it can modulate the proliferation and metastasis of colorectal cancer cells. It function as the ceRNA to regulate the expression of DVL3 by sponging miR-29a-3p.

\section{Introduction}

Colorectal cancer (CRC) is a common malignancy of digestive tract. The morbidity of CRC ranks third in the diagnosed malignancies, and mortality of CRC ranks fifth $[1,2]$. At present, the treatment methods and approaches of CRC are limited. The main methods are surgery and chemotherapy, but postoperative recurrence and metastasis can reach to $40 \%-50 \%$ [3-5], the survival rate and quality of life of patients are expected to be improved. Exploring the mechanisms of the carcinogenesis and progression of CRC is important for improving the prognosis of CRC patients.

Long-chain non-coding RNA (IncRNA) is a class of RNA molecules that are more than 200 nucleotides in length but cannot encode proteins. It regulates gene expression at the transcriptional, post-transcriptional, and epigenetic levels, thereby affecting cell differentiation, metabolism, proliferation and apoptosis [6]. In the development of some human cancers, the expression of genes on the $Y$ chromosome plays an important role [7-10]. LncRNA testis-specific transcript, Y-linked 15 (TTTY15) is located in the AZFa region of the $Y$ chromosome, and a recent study shows that the expression of TTTY15 is up-regulated in prostate cancer tissues, and it can drive the progression of prostate cancer tissues by sponging let-7 [11]. Nevertheless, the expression and function of TTTY15 in CRC are much less explored. 
MicroRNA (miRNA) is a kind of non-coding RNA (ncRNA) of about 22 nucleotides in length [12]. The researches reported recently illustrated that the differential expression of miR-29a-3p is related to the carcinogenesis and progression of diverse cancers. It is down-regulated in liver cancer, and its low expression is associated with the lower survival rate of patients; moreover, overexpression of miR-29a-3p can inhibit the proliferation and migration of liver cancer cells by targeting IGF1R [13]. The expression of miR-29a-3p is also significantly down-regulated in gastric cancer and its low expression facilitate the proliferation, migration and invasion of cancer cells [14]. There are also studies on the expression and mechanism of miR-29a-3p in CRC $[15,16]$, but the research is still not perfect, and its upstream and downstream molecular mechanisms still need to be further studied.

Disheveled (DVL), a positive regulator of the Wnt signaling pathway, which is located upstream of $\beta$ catenin and highly expressed in a variety of tumors. Wnt signaling pathway is the key pathway to maintain stem cell-like characteristics such as stem cell growth and self-renewal. Its abnormal activation or mutation is closely related to tumorigenesis $[18,19]$. The carcinogenesis and progression of CRC are accompanied by activation of the Wnt pathway $[20,21]$. DVL is widely present in the cytoplasm, and DVL3 is a member of its family, but the current molecular mechanism of DVL3 in CRC is expected to be explored further.

This study mainly explored the expression and function of TTTY15 in CRC, and the existence and role of TTTY15/miR-29a-3p/DVL3 axis in CRC were confirmed as well.

\section{Materials And Methods}

\subsection{Tissue Specimens}

The tumor tissues and normal paracancerous tissues of 51 male patients who underwent radical resection of CRC at Linyi Central Hospital from April 2017 to April 2018 were randomly collected. None of patients received chemotherapy or radiotherapy before surgery. The control group specimens were resected from normal paracancerous tissues of the same patient (at least $2 \mathrm{~cm}$ from the surgical margin), and no cancer cells were found during pathological examination. The collection and use of patient tissue samples were approved by the Linyi Central Hospital Ethics Committee. All patients involved gave informed consent to the study and signed a written consent form. Tissue samples were surgically removed and immediately stored at $-196^{\circ} \mathrm{C}$ in liquid nitrogen until use.

\subsection{Cell culture}

Human CRC cell line (HCT-116, HT29, SW480, SW620, DLD-1 cells) and normal colonic epithelial cell line CCD841 were purchased from the Institute of Biochemistry and Cell Biology of the Chinese Academy of Sciences (Shanghai, China). The cells were cultured in RPMI-1640 medium (Gibco, Carlsbad, CA, USA) containing 10\% fetal bovine serum (FBS; Gibco, Carlsbad, CA, USA) and $100 \mathrm{U} / \mathrm{mL}$ penicillin/streptomycin (Life Technologies, USA) in a constant temperature incubator at $37{ }^{\circ} \mathrm{C}$ in $5 \% \mathrm{CO}_{2}$. The solution was 
changed every $3 \mathrm{~d}$, and the cells were passaged when the bottom of the culture flask was covered with the cells. Cells in the logarithmic growth were selected for assays.

\subsection{Cell transfection}

The cells in the logarithmic growth phase were inoculated into a 6-well plate with $2.5 \times 10^{5}$ cells per well and the cells were transfected according to the instructions of the Lipofectamine ${ }^{8} 3000$ (Invitrogen; ThermoFisherScientific, Inc., USA). TTTY15 shRNA, TTTY15 overexpression plasmid, miR-29a-3p mimics, miR-29a-3p inhibitor, empty vectors, microRNA mimics control were transfected into cells respectively. After $48 \mathrm{~h}$ of culture, RNA was extracted to verify the transfection efficiency.

\subsection{RNA isolation and quantitative real-time polymerase chain reaction (qRT-PCR)}

Total RNA was extracted and purified from tissues and cell lines according to the instructions of TRIzol reagent (Invitrogen, Carlsbad, USA). RNA purity and concentration were measured using the NanoDrop ND-2000 spectrophotometer (NanoDrop Wilmington DE). M-MLV Reverse Transcriptase (Thermo Fisher Scientific, Inc., Rockford, IL, USA) containing $5 \mu \mathrm{g}$ of total RNA was used to reverse transcribed to cDNA. qRT-PCR was performed on the ABI 7300 machine (Applied Biosystems, Foster City, CA, USA) with SYBR Green PCR Master Mix (Thermo Fisher Scientific, Inc.). The qRT-PCR conditions were as follows: predenaturation at $95^{\circ} \mathrm{C}$ for $10 \mathrm{~min}, 95^{\circ} \mathrm{C}$ for $15 \mathrm{~s}, 60^{\circ} \mathrm{C}$ for $15 \mathrm{~s}, 45$ cycles, and the fluorescence signal temperature was obtained at $60^{\circ} \mathrm{C}$. The relative expression of TTTY15, miR-29a-3p and DVL3 was calculated by the $2^{-\triangle \Delta C t}$ method using GAPDH and $U 6$ as internal reference. Primers were listed in Table 1.

\subsection{Cell counting kit-8 (CCK-8) assay}

The cell proliferation curve was determined using CCK-8 assay. HCT-116 and SW480 cells (3000 cells per well) were seeded in 96-well plates and incubated for 1, 2, 3, and $4 \mathrm{~d}$, respectively. Subsequently, $10 \mu \mathrm{L}$ of enhanced Cell Counting Kit-8 (Beyotime, Beijing, China) was added and the cells were incubated at $37^{\circ} \mathrm{C}$ for $1 \mathrm{~h}$. Then then culture was terminated and the absorbance value (OD value) of each well was measured at $450 \mathrm{~nm}$ on a microplate reader, and the relative OD ratio was used to indicate the cell proliferation.

\subsection{Plate colony formation assay}

Cells in the logarithmic growth phase were trypsinized with $0.25 \%$ trypsin and dispersed into single cells and counted. The cell concentration was adjusted to 1000 cells per dish, and the cell suspension was inoculated separately into a dish containing $3 \mathrm{~mL}$ of $37^{\circ} \mathrm{C}$ pre-warmed culture solution and gently rotated to evenly disperse the cells. Subsequently, the cells were cultured in an environment of $37{ }^{\circ} \mathrm{C}, 5 \% \mathrm{CO}_{2}$ and saturated humidity for 2 to 3 weeks. The number of cells contained in each cell clone was counted, and the cell colony formation rate was calculated and photographed. Clonal formation rate $=$ number of clones/number of cells inoculated $* 100 \%$.

\subsection{Tranwell assay}


Transwell assay was used to detect the migration and invasion. Matrigel was used in invasion assay, but not in migration assay. The cells in each group transfected for $48 \mathrm{~h}$ were taken, trypsinized, resuspended with serum-free medium, counted and seeded into the upper chamber, which was not used in the migration assay. RPMI-1640 medium containing 10\% FBS was added to the lower chamber. The cells were placed in a $37^{\circ} \mathrm{C}, 5 \% \mathrm{CO}_{2}$ incubator and cultured for $24 \mathrm{~h}$. After that, the cells that failed to migrate or invade were removed from the upper chamber. After being fixed for 4 minutes with $4 \%$ paraformaldehyde, the migrated or invaded cells were stained with $0.5 \%$ crystal violet. After being rinsed off by the tap water, the cells were observed under an inverted microscope, and cell counts were performed using five fields of view, and the mean value was taken.

\subsection{Flow cytometry}

Cellular apoptosis was detected and analyzed by flow cytometry. Cells in the logarithmic growth phase were washed twice with PBS, fixed in $70 \%$ ethanol, and stored at $4{ }^{\circ} \mathrm{C}$ overnight. Then the cells were washed once with PBS, and the cell density was adjusted to $1 \times 10^{6} \mathrm{cells} / \mathrm{mL}$. The cells were then doublestained with AnnexinV-FITC and Propidium iodide and incubated for 15 minutes in the dark at room temperature. Finally, the stained cells were analyzed using flow cytometry (BD Biosciences).

\subsection{Dual-luciferase reporter gene assay}

Luciferase reporter gene assay was performed using the dual-luciferase reporter assay system (Promega, Madison, WI, USA). The target fragment of wild type TTTY15 and mutant TTTY15 was constructed and integrated into pGL3 vector (Promega, Madison, WI, USA) to construct pGL3-TTTY15-wild type (TTTY15wt and pGL3-TTTY15-mutant (TTTY15-mut) reporter vector. Then TTTY15-wt or TTTY15-mut was cotransfected with miR-29a-3p mimics or control microRNA mimics. After $48 \mathrm{~h}$ of transfection, luciferase activity was determined in accordance with the manufacturer's instructions. All experiments were performed in triplicate and repeated three times. The same method was used to construct DVL3-wt and DVL3-mut, and to detect the targeted binding relationship between miR-29a-3p and the 3'UTR of DVL3.

\subsection{Western Blot}

The RIPA lysis buffer (Beyotime Biotechnology, Beijing, China) containing PMSF was used to extract the protein sample. Protein samples were added for SDS-PAGE and transferred to the nitrocellulose membrane. After being blocked with $5 \%$ fat-free milk, the membrane was incubated with primary antibody anti-DVL3 (dilution 1:1000, Cell Signaling Technology) and anti-GAPDH (dilution 1:2000, Santa Cruz) antibody at room temperature for $8 \mathrm{~h}$. After being washed, the membrane was incubated with horseradish peroxidase (HRP) conjugated secondary antibody (1:2000, Santa Cruz Biotechnology) for $1 \mathrm{~h}$ at room temperature. Ultimately, The membrane was placed on an automatic developing device (ChemiDocXRS imaging system) to calculate the gray value.

\subsection{Statistical analysis}


Data analysis was performed using SPSS18.0 statistical software. All data were expressed as mean \pm SD. Statistical analysis was performed by student's t-test. Pearson assay was employed to analyze the correlation among TTTY15, miR-29a-3p and DVL3 expression. $P<0.05$ was statistically significant.

\section{Result}

\subsection{TTTY15 was highly expressed in CRC tissues and cell lines.}

In order to explore the expression of TTTY15 in CRC, qRT-PCR assay was employed to detect TTTY15 expression in CRC tissues, paracancerous tissues and CRC cell lines (HCT-116, HT29, SW480, SW620, DLD-1 cells) and normal colorectal epithelial cell line (CCD841 cells). The results revealed that TTTY15 was significantly up-regulated in CRC tissues and cell lines compared non-cancerous tissues and cell line (Fig. 1A \& B). To explore the significance of high expression of TTTY15 in CRC, we divided the patients into high-expression and low-expression groups according to the expression level of TTTY15. The results implied that the high expression of TTTY15 was significantly associated with lymph node metastasis in CRC (Tab. 2).

\subsection{TTTY15 enhanced proliferation, invasion and migration of CRC cells and impeded the apoptosis.}

In order to investigate the role of TTTY15 high expression in CRC, we overexpressed TTTY15 in HCT-116 cell line and knocked down TTTY15 in HT29 cell line (Fig. 2A). The cell proliferation was verified by CCK-8 assay and plate colony formation assay. The results showed that the proliferation of HCT-116 cells was significantly increased after TTTY15 overexpression, and the proliferation of HT29 cells was decreased after TTTY15 knockdown (Fig. 2B\&C\&D). In addition, the metastasis of cancer was related to the migration and invasion of cancer cells, so the cell invasion and migration were detected by Transwell assay, and the results showed that the invasion and migration of cells increased after overexpression of TTTY15. Consistently, there was also a decrease in the invasion and migration of HT29 cells after knockdown of TTTY15 (Fig. 2E\&F). After that, flow cytometry was employed to detect cell apoptosis. The results indicated that the apoptosis of HCT-116 cells was significantly decreased after TTTY15 overexpression, while the apoptosis of HT-29 cells was increased after TTTY15 knockdown (Fig. 2G).

\subsection{TTTY15 directly targeted miR-29a-3p and reduced its expression in CRC.}

To further explore the downstream molecular mechanisms of TTTY15, we conducted a bioinformatics analysis by querying the LncBase Predicted v.2 database (http://carolina.imis.athena-innovation.gr) and found that there were two potential binding sites between TTTY15 and miR-29a-3p (Fig. 3A). We further verified them by dual-luciferase activity assay. The results showed that miR-29a-3p mimics decreased the luciferase activity of wild type TTTY15 sequences. However, the effect of miR-29a-3p on mutant TTTY15 sequences was not significant (Fig. 3B). In addition, we analyzed the correlation between TTTY15 expression and miR-29a-3p expression in CRC tissues, and the results showed a significant negative correlation between the them (Fig. $3 C, r^{2}=0.513$ ). Previous studies have reported that miR-29a-3p is lowexpressed in CRC, and its low expression can enhance cancer cell proliferation and invasion, so we 
suspected that TTTY15 functioned as the upstream molecules of miR-29a-3p in CRC. In vitro cell experiments showed that the expression of miR-29a-3p was down-regulated after overexpression of TTTY15, and the expression of miR-29a-3p was up-regulated after knockdown of TTTY15 (Fig. 3D). However, regulating the expression of miR-29a-3p had no significant effect on the expression of TTTY15 (Fig. 3E).

\section{4 miR-29a-3p targeted and down-regulated DVL3 in CRC.}

Recently, studies have confirmed that DVL3 was a direct target of the miR-29 family. By querying StarBase database (http://starbase.sysu.edu.cn) found that there was a binding site between miR-29a-3p and DVL3 (Fig. 4A). Therefore, we speculated that miR-29a-3p could regulate DVL3 and participate in the development of CRC. As expected, miR-29a-3p mimics decreased the luciferase activity of wild type DVL3 3'UTR, but had no significant effect on mutant DVL3, which confirmed the binding relationship (Fig. 4B). Additionally, miR-29a-3p was significantly negatively correlated with DVL3 expression (Fig. 4C, $\mathrm{r}^{2}=0.570$ ), and TTTY15 and the expression of DVL3 showed a significant positive correlation (Fig. 4D, $\mathrm{r}^{2}=0.568$ ). After that, we found in in vitro cell experiments that the expression of DVL3 was significantly decreased after overexpression of miR-29a-3p, whereas the expression of DVL3 was remarkably increased after inhibition of miR-29a-3p. What's more, DVL3 was increased after overexpression of TTTY15 and decreased after knocking down TTTY15 (Fig. 4E-F). Collectively, we confirmed that DVL3 was modulated by TTTY15/miR-29a-3p axis in CRC.

\subsection{TTTY15 knockdown repressed CRC cell viability, migration, and invasion but induced cell apoptosis via regulating miR-29a-3p.}

Based on the above findings, we made a hypothesis that there could be a TTTY15/miR-29a-3p/DVL3 axis during CRC progression, so we then constructed TTTY15 knockdown cells and TTTY15 shRNA/miR-29a$3 p$ inhibitor co-transfection cells with HT29 cells. The expression of TTTY15, miR-29a-3p and DVL3 in different groups of cells was detected by qRT-PCR and western blot (Fig. 5A \& B \& C). The results of CCK8 assay and plate colony formation assay revealed that the cell proliferation in TTTY15 shRNA/miR-29a$3 p$ inhibitor group was higher than that in TTTY15 knockdown group, suggesting miR-29a-3p inhibitor transfection reversed the attenuated cell proliferation induced by TTTY15 knockdown (Fig. 5D\&E). Similarly, the results of Transwell assay implied that the cell invasion and migration in TTTY15 knockdown group were significantly lower than that in TTTY15 shRNA/miR-29a-3p inhibitor group (Fig. $5 F \& G$ ). The results of flow cytometry analysis showed that miR-29a-3p inhibitor transfection could effectively reduce the increase of apoptosis caused by TTTY15 knockdown (Fig. 5H).

\section{Discussion}

CRC is a common malignancy. Because early symptoms are not obvious, a large number of patients have metastatic CRC when they are diagnosed and lost the opportunity for surgical treatment. The main therapeutic methods for patients with metastatic CRC are chemotherapy, and molecular targeting therapy, 
but the therapeutic effect is still not satisfactory $[22,23]$. Exploring the molecular mechanism of CRC and finding feasible molecular therapeutic targets are important for improving patient survival.

Male and female have different susceptibility to different cancers, and their clinical outcomes and prognosis are different. $Y$ chromosome has a specific effect on male's normal development, physiology and disease [24]. Studies have reported that the morbidity of CRC in males is significantly higher than that of females, which is associated with higher levels of estrogen in females [25]. Nonetheless, the impact of changes in gene expression on the $Y$ chromosome on the development of CRC remains largely unknown. TTTY15 is located on the Y chromosome. It has been reported that the expression of TTTY15 is up-regulated in myocardial infarction; down-regulation of TTTY15 expression can inhibit hypoxiainduced cardiomyocyte apoptosis [26]. It has also been reported that the expression of TTTY15 is closely related to the carcinogenesis of prostate cancer. It can be fused with USP9Y, and TTTY15-USP9Y fusion is confirmed as a biomarker for early detection of prostate cancer [27]; TTTY15 is up-regulated in PC tissues, and down-regulation of its expression can inhibit the development of tumors [11]. Our study first explored the expression and role of TTTY15 in CRC, and confirmed its oncogenic effect. To some extent, the gender differences in CRC are explained, providing a new therapeutic target for the treatment of this disease.

Accumulating reports imply that miRNAs are usually dysregulated in CRC and function as a tumor suppressor or oncogenic factor. For instance, miR-296 is down-regulated in CRC tissues and cells, and its low expression can enhance the invasion and migration of CRC cells, and miR-296 can target S100A4 to inhibit epithelial-mesenchymal transition (EMT) in CRC [28]; miR-18b expression is remarkably increased in CRC tissues, and its overexpression promotes cancer cell proliferation by facilitating cell cycle progression by targeting CDKN2B [29]. In recent years, several studies have found that IncRNAs can regulate miRNA expression levels by targeting it and thus affect tumor progression [30, 31]. To explore the downstream molecular mechanism of TTTY15 in CRC, we identified the binding relationship between TTTY15 and miR-29a-3p. It should be noted that the role of miR-29a-3p in CRC has also been reported. Down-regulation of miR-29a-3p can promote the invasion and migration of CRC cells, and it can repress the invasion and migration of cancer cells by reducing the expression of CDC42BPA [16]. We observed that in CRC tissues, the expression of TTTY15 and miR-29a-3p was notably negatively correlated. And it was found by in vitro cell experiments that the expression of miR-29a-3p was significantly increased after knocking down TTTY15; the expression of miR-29a-3p was significantly decreased after overexpression of TTTY15. These data not only explained the mechanism by which TTTY15 participated in CRC progression, but also help clarify the reason of dysregulation of miR-29a-3p in CRC.

Additionally, previous studies have reported that in the osteoarthritis, the miR-29 family can negatively regulate the signaling pathways of Wnt, Smad, and NF-KB signaling, thereby affecting the progression of the disease, while DVL3, FZD3, FZD5, FRAT2, and CK2A2 have been verified to be the direct targets of the miR-29 family [32]. The activation of Wnt signaling pathway is involved in the development of CRC, after taking the above findings into consideration, we made a hypothesis that there may be TTTY15/miR-29a3p/DVL3 axis in CRC. In this work, luciferase assay was employed to verify the binding relationship 
between miR-29a-3p and the 3'UTR of DVL3. Furthermore, the effect of changes in the expression of TTTY15 and miR-29a-3p on the expression of DVL3 was further examined by rescue experiments, and it was confirmed that the transfection of miR-29a-3p mimics decreased the increase of proliferation, migration and invasion of cancer cells caused by overexpression of TTTY15. The above results confirmed the existence of the TTTY15/miR-29a-3p/DVL3 axis in CRC.

\section{Conclusion}

In summary, TTTY15 expression is significantly up-regulated in CRC, and can enhance DVL3 expression via regulating miR-29a-3p and facilitate the development of CRC.The results of this study further deepen the study of the molecular mechanisms of CRC and provide new clues for its treatment.

\section{Declarations}

Ethics approval and consent to participate: The collection and use of patient tissue samples were approved by the Linyi Central Hospital Ethics Committee. All patients involved gave informed consent to the study and signed a written consent form.

Consent for publication: All the authors reviewed the final edition of this manuscript and agreed to submit.

Availability of data and materials: The data used to support the findings of this study are available from the corresponding author upon request.

Competing interests: The authors declare that they have no competing interest.

Funding: None.

Authors' contributions: Conceived and designed the experiments: YFL; performed the experiments: MZC, YB; statistical analysis: MZC, YB; Wrote the paper: MZC, YB.

Acknowledgement: None.

\section{References}

1. Siegel RL, Miller KD, Jemal A. Cancer statistics, 2016. Ca Cancer J Clin. 2016, 66 (1): 7-30.

2. Chen W, Zheng R, Zhang S, et al. Cancer incidence and mortality in China, 2013. Cancer Lett. 2017 Aug 10; 401: 63-71.

3. Zafar SY, Malin JL, Grambow SC, et al. Chemotherapy use and patient treatment preferences in advanced colorectal cancer: a prospective cohort study. Cancer. 2013, 119 (4): 854-62.

4. Altomare DF, Di Lena M, Porcelli F, et al. Exhal ed volatile organic compounds identify patients with colorectal cancer. Br J Surg. 2013, 100 (1): 144-50. 
5. Jianping Sheng, Liqiang Wang, Yajing Han, Wansong Chen, Hong Liu, Min Zhang, Liu Deng, and YouNian Liu, Dual Roles of Protein as a Template and a Sulfur Provider: A General Approach to Metal Sulfides for Efficient Photothermal Therapy of Cancer. Small. 2018, 14 (1): 1702529.

6. Dhamija S, Diedericha S. From junk to master regulators of invasion: IncRNA functions in migration, EMT and metastasis. Int J Cancer. 2016 Jul 15; 139 (2): 269-80.

7. Wright DJ, Day FR, Kerrison ND, et al. Genetic variants associated with mosaic $Y$ chromosome loss highlight cell cycle genes and overlap with cancer susceptibility. Nat Genet. 2017 May; 49 (5): 674679.

8. Kido T, Lau YF. Roles of the Y chromosome genes in human cancers. Asian J Androl. 2015 May-Jun; 17 (3): 373-80.

9. Patel R, Khalifa AO, Isali I, et al. Prostate cancer susceptibility and growth linked to Y chromosome genes. Front Biosci (Elite Ed). 2018 Mar 1; 10: 423-436.

10. Wallrapp C, Hähnel S, Boeck W, et al. Loss of the Y chromosome is a frequent chromosomal imbalance in pancreatic cancer and allows differentiation to chronic pancreatitis. Int J Cancer. 2001 Feb 1; 91 (3): 340-4.

11. Xiao G, Yao J, Kong D, et al. The Long Noncoding RNA TTTY15, Which Is Located on the $Y$ Chromosome, Promotes Prostate Cancer Progression by Sponging let-7. Eur Urol. 2018 Dec 7. pii: S0302-2838 (18) 30869-8.

12. Vishnoi A, Rani S. MiRNA Biogenesis and Regulation of Diseases: An Overview. Methods Mol Biol. 2017; 1509: 1-10.

13. Wang X, Liu S, Cao L, et al. miR-29a-3p suppresses cell proliferation and migration by downregulating IGF1R in hepatocellular carcinoma. Oncotarget. 2017 Sep 23; 8 (49): 86592-86603.

14. Zhao Z, Wang L, Song W, et al. Reduced miR-29a-3p expression is linked to the cell proliferation and cell migration in gastric cancer. World J Surg Oncol. 2015 Mar 12; 13: 101. doi: 10.1186/s12957-0150513-х.

15. Kara M, Yumrutas O, Ozcan O, et al. Differential expressions of cancer-associated genes and their regulatory miRNAs in colorectal carcinoma. Gene. 2015 Aug 1; 567 (1): 81-6.

16. He PY, Yip WK, Chai BL, et al. Inhibition of cell migration and invasion by miR-29a-3p in a colorectal cancer cell line through suppression of CDC42BPA mRNA expression. Oncol Rep. 2017 Dec; 38 (6): 3554- 3566.

17. Huang S, Chen J, Tian R, et al. Down-regulation of dishevelled-2 inhibits cell proliferation and invasion in hepatoblastoma. Pediatr Blood Cancer. $2018 \mathrm{Jul} ; 65$ (7): e27032.

18. Pai VC, Hsu CC, Chan TS, et al. ASPM promotes prostate cancer stemness and progression by augmenting Wnt-Dvl-3- $\beta$-catenin signaling. Oncogene. 2019 Feb; 38 (8): 1340-1353.

19. Zou YF, Xie CW, Yang SX, et al. AMPK activators suppress breast cancer cell growth by inhibiting DVL3-facilitated Wnt/ $\beta$-catenin signaling pathway activity. Mol Med Rep. 2017 Feb; 15 (2): 899-907. 
20. Basu S, Haase G, Ben-Zeev A. Wnt signaling in cancer stem cells and colon cancer metastasis. F1000Res. 2016 Apr 19;5. pii: F1000 Faculty Rev-699.

21. Schatoff EM, Leach BI, Dow LE. Wnt Signaling and Colorectal Cancer. Curr Colorectal Cancer Rep. 2017 Apr; 13 (2): 101-110.

22. Zafar SY, Malin JL, Grambow SC, et al. Chemotherapy use and patient treatment preferences in advanced colorectal cancer: a prospective cohort study[J]. Cancer, 2013 Feb 15; 119 (4): 854-62.

23. Finnberg N, Gokare P, El-Deiry WS. Novel and Emerging Targeted Therapies of Colorectal Cancer. Curr Clin Pharmacol. 2015; 10 (4): 279-98.

24. Wright DJ, Day FR, Kerrison ND, et al. Genetic variants associated with mosaic Y chromosome loss highlight cell cycle genes and overlap with cancer susceptibility. Nat Genet. 2017 May; 49 (5): 674679.

25. Foster PA. Oestrogen and colorectal cancer: Mechanisms and controversies. Int J Colorectal Dis. 2013 Jun; 28 (6): 737-49.

26. Huang S, Tao W, Guo Z, et al. Suppression of long noncoding RNA TTTY15 attenuates hypoxiainduced cardiomyocytes injury by targeting miR-455-5p. Gene. 2019 Jun 15; 701: 1-8.

27. Zhu Y, Ren S, Jing T, et al. Clinical utility of a novel urine-based gene fusion TTTY15-USP9Y in predicting prostate biopsy outcome. Urol Oncol. 2015 Sep; 33 (9): 384.e9-20.

28. He Z, Yu L, Luo S, et al. miR-296 inhibits the metastasis and epithelial-mesenchymal transition of colorectal cancer by targeting S100A4. BMC Cancer. 2017 Feb 16; 17 (1): 140.

29. Li Y, Chen M, Liu J, et al. Upregulation of MicroRNA 18b Contributes to the Development of Colorectal Cancer by Inhibiting CDKN2B. Mol Cell Biol. 2017 Oct 27; 37 (22).

30. Li X, Deng SJ, Zhu S, et al. Hypoxia-induced IncRNA-NUTF2P3-001 contributes to tumorigenesis of pancreatic cancer by derepressing the miR-3923/KRAS pathway. Oncotarget. 2016 Feb 2; 7 (5): 600014.

31. Wei W, Liu Y, Lu Y, et al. LncRNA XIST Promotes Pancreatic Cancer Proliferation Through miR133a/EGFR. J Cell Biochem. 2017 Oct; 118 (10): 3349-3358.

32. Le LT, Swingler TE, Crowe N, et al. The microRNA-29 family in cartilage homeostasis and osteoarthritis. J Mol Med (Berl). 2016 May; 94 (5): 583-96.

\section{Tables}

Table 1. Primers used in this study. 


\begin{tabular}{|c|c|}
\hline Name & Primer sequences $\square 5^{\prime}-3^{\prime} \square$ \\
\hline \multirow[t]{2}{*}{ TTTY15 } & Forward:TCTATGACCTGGAAGC \\
\hline & Reverse:ATCTGATG GAACCCTA \\
\hline \multirow[t]{2}{*}{ miR-29a-3p } & Forward:AGCACCAUCUGAAAUCGGUUA \\
\hline & Reverse:GTGCAGGGTCCGAGGT \\
\hline \multirow[t]{2}{*}{ DVL3 } & Forward:CCATCACCAGCTCCATCC \\
\hline & Reverse:CTGCTGCGTGTAGTGTGG \\
\hline \multirow[t]{2}{*}{ GAPDH } & Forward:ACAACTTTGGTATCGTGGAAGG \\
\hline & Reverse:GCCATCACGCCACAGTTTC \\
\hline \multirow[t]{2}{*}{ U6 } & Forward:CTCGCTTCGGCAGCACA \\
\hline & Reverse:AACGCTTCACGAATTTGCGT \\
\hline
\end{tabular}




\begin{tabular}{|c|c|c|c|c|c|}
\hline \multirow[t]{3}{*}{ Clinicopathologic factors } & \multirow{3}{*}{$\begin{array}{l}\text { Patients number } \\
\qquad(\mathrm{n}=51)\end{array}$} & \multicolumn{2}{|c|}{ TTTY15 expression } & \multirow[t]{3}{*}{$\mathrm{X}^{2}$} & \multirow[t]{3}{*}{$p$} \\
\hline & & Lower & Higher & & \\
\hline & & $(n=23)$ & $(\mathrm{n}=28)$ & & \\
\hline Age & & & & 0.126 & 0.723 \\
\hline$\leq 60$ & 28 & 12 & 16 & & \\
\hline$>60$ & 23 & 11 & 12 & & \\
\hline Location of the tumor & & & & 0.216 & 0.642 \\
\hline Colon & 24 & 10 & 14 & & \\
\hline Rectum & 27 & 13 & 14 & & \\
\hline Diameter $\llbracket \mathrm{cm} \rrbracket$ & & & & 0.479 & 0.489 \\
\hline$<5$ & 25 & 10 & 15 & & \\
\hline$\geq 5$ & 26 & 13 & 13 & & \\
\hline TNM stage & & & & 2.753 & 0.097 \\
\hline I-II & 44 & 21 & 23 & & \\
\hline III-IV & 7 & 1 & 6 & & \\
\hline Lymphatic metastasis & & & & 26.609 & $<0.001^{* * *}$ \\
\hline Yes & 29 & 4 & 25 & & \\
\hline No & 22 & 19 & 3 & & \\
\hline
\end{tabular}

* $p<0.05$

Figures 
A

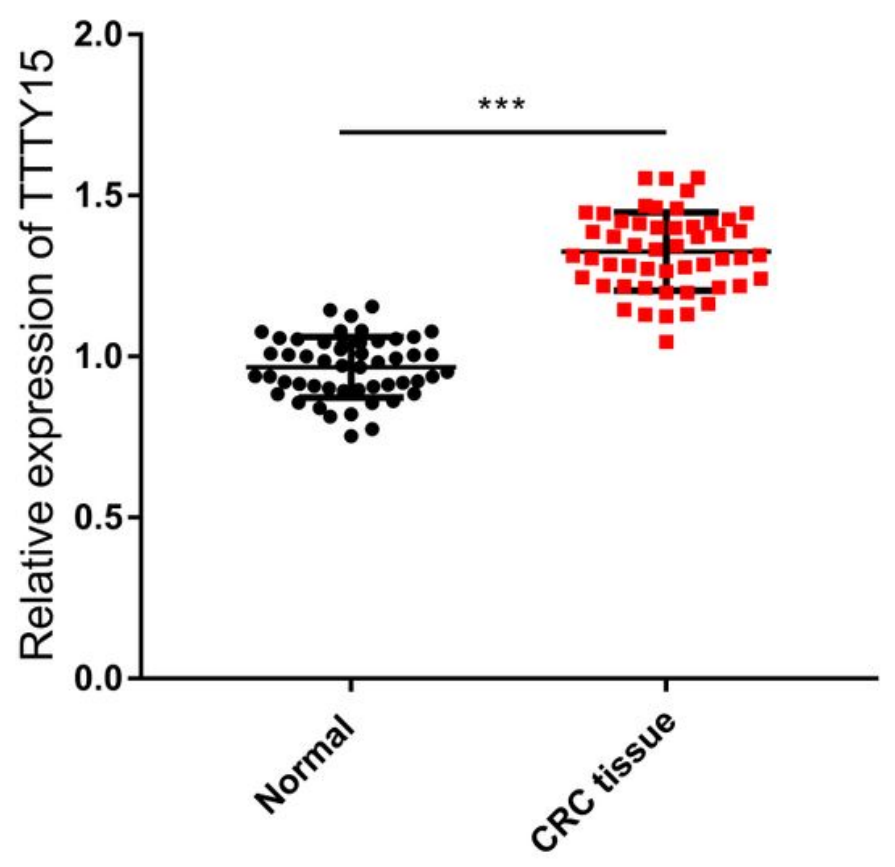

B

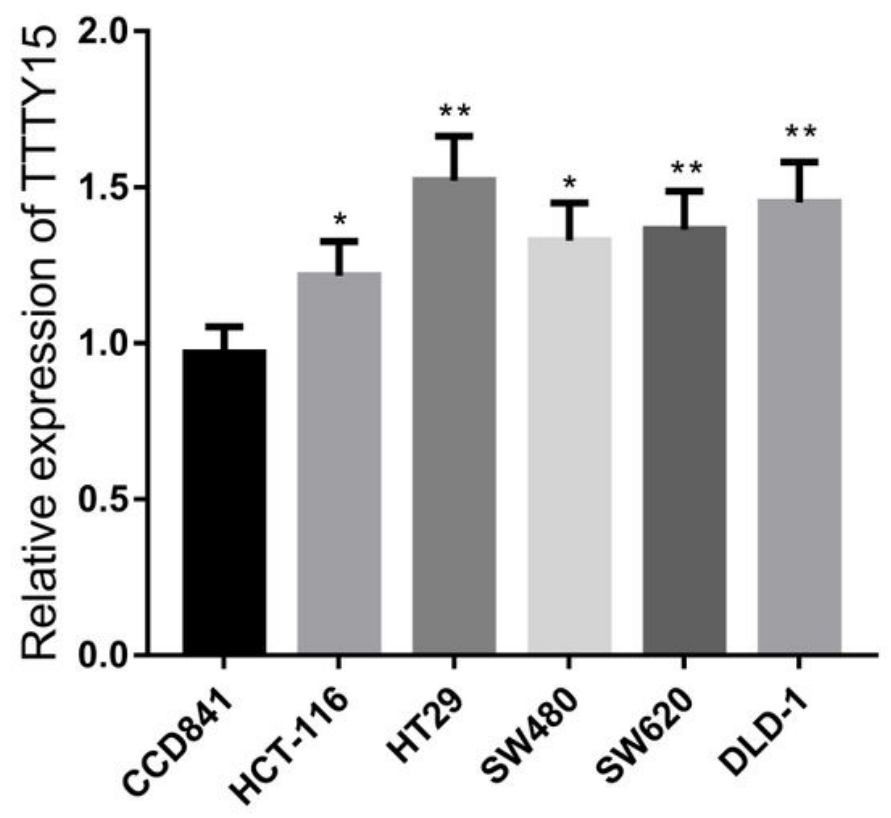

Figure 2

Expression of TTTY15 in CRC tissues and cell lines A. The qRT-PCR assay was employed to detect the expression of TTTY15 in 51 pairs of CRC tissues and paracancerous tissues. B. qRT-PCR assay was employed to detect the expression of TTTY15 in CRC cell lines (HCT-116, HT29, SW480, SW620, DLD-1 cells) and normal colorectal epithelial cell line CCD841. (Compared with Normal group or CCD841, * indicates $p<0.05$, ** indicates $p<0.01$, *** indicates $p<0.001$ ) 
A

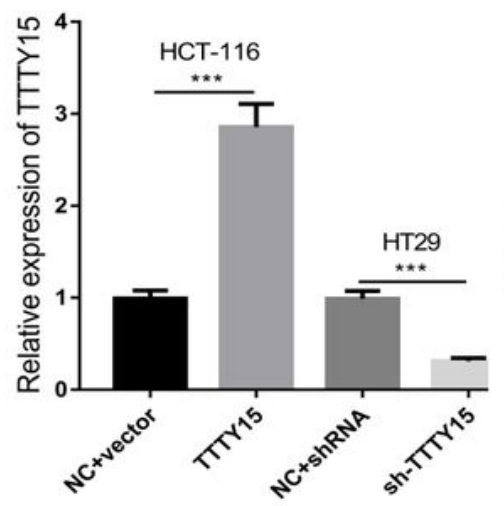

$\mathrm{D}$
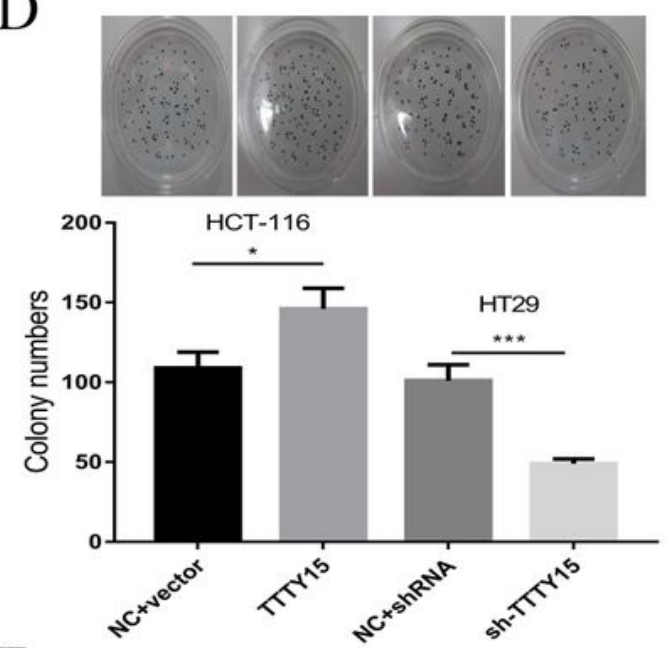

$\mathrm{F}$
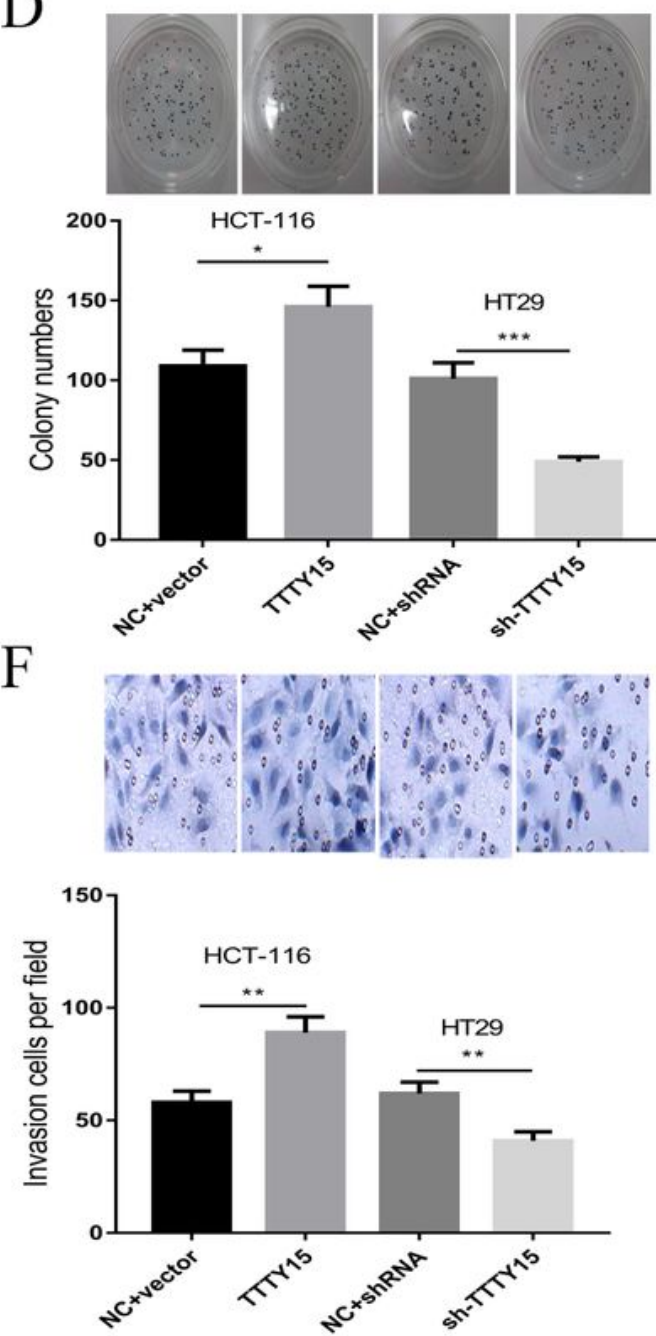

B

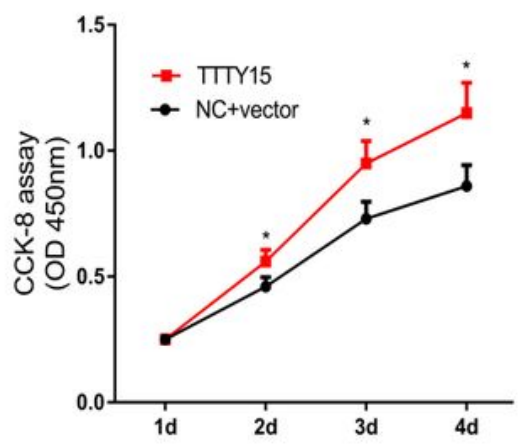

$\mathrm{E}$
$\mathrm{C}$

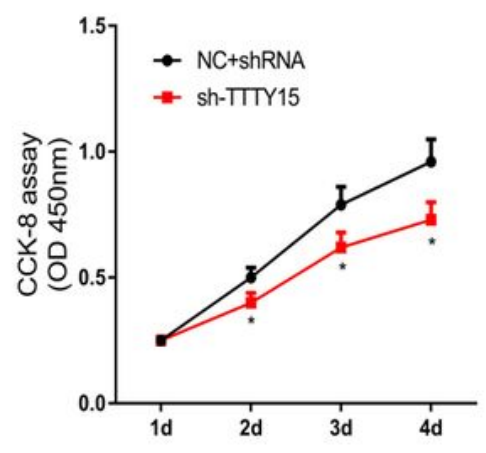

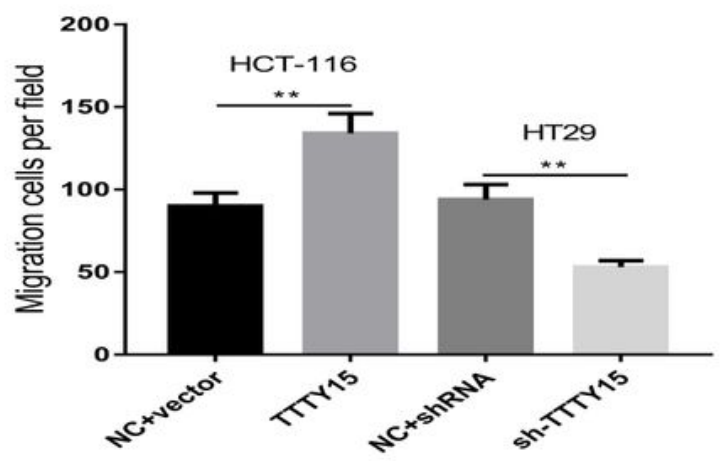

G

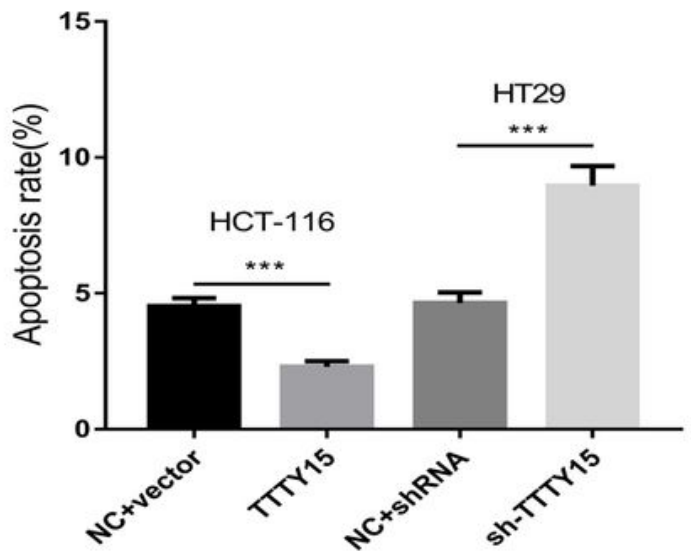

Figure 4

TTTY15 promotes the proliferation, invasion and migration of CRC cells and inhibits the apoptosis $\mathrm{A}$. The expression levels of TTTY15 in HCT-116 and HT29 cells after overexpression and knockdown of TTTY15 were detected by qRT-PCR. B\&C\&D. CCK-8 assay and plate colony formation assay were employed to detect the proliferation of TTTY15 overexpression and knockdown cells. E\&F. The Transwell assay was employed to detect the migration and invasion of cells after overexpression and knockdown of TTTY15. 
G. Flow cytometry was used to detect the cell apoptosis of TTTY15 after overexpression and knockdown. (Compared with $\mathrm{NC}+$ vector or NC + shRNA, * means indicates $\mathrm{p}<0.05$, ** indicates $\mathrm{p}<0.01$, *** indicates $p<0.001)$

A

TTTY15 wt :AGAAGAACAGCACCAAGGGU AUGGUGCUG
miR-29a-3p: UUGG CUAAAGUCUACCACGAU
TTTY15-mut:AGAAGAACAGCAGUAAGAC CGCCAUAUG

TTTY15 wt :UGGGACGUUUACCUGAG CGCUUGGUGCUG miR-29a-3p: $\quad$ UUGG CUAAAGUCUACCACGAU TTTY15-mut:UGGGACGUUUUGCUUAUG GGCUAUCGCCGG
C

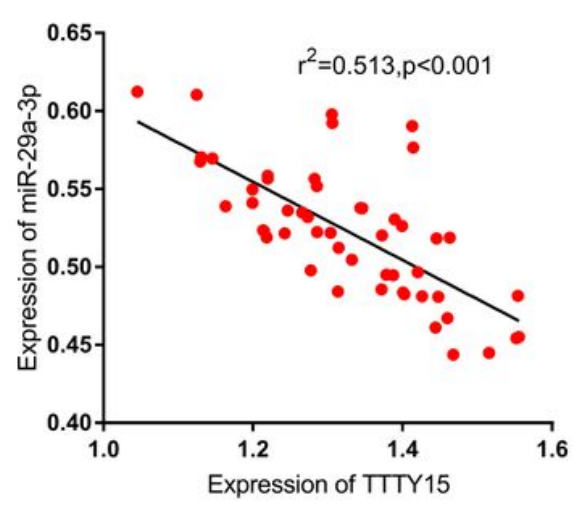

D

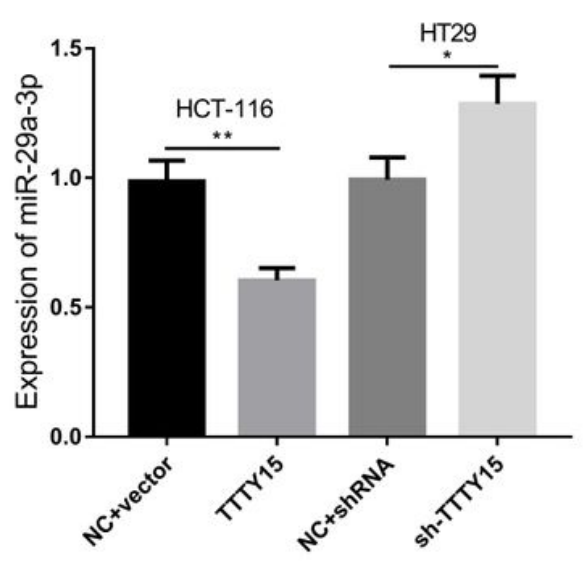

B

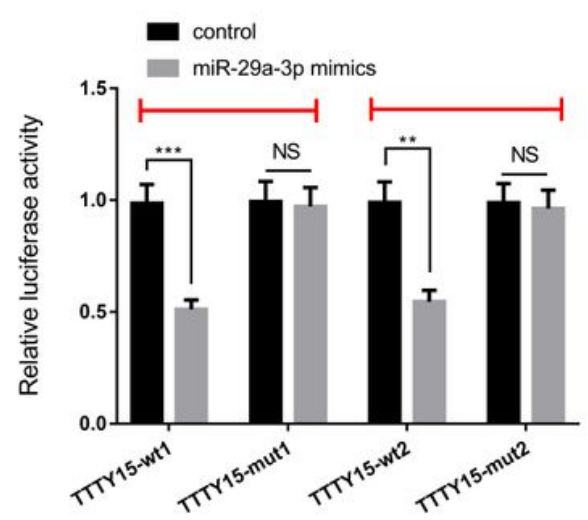

E

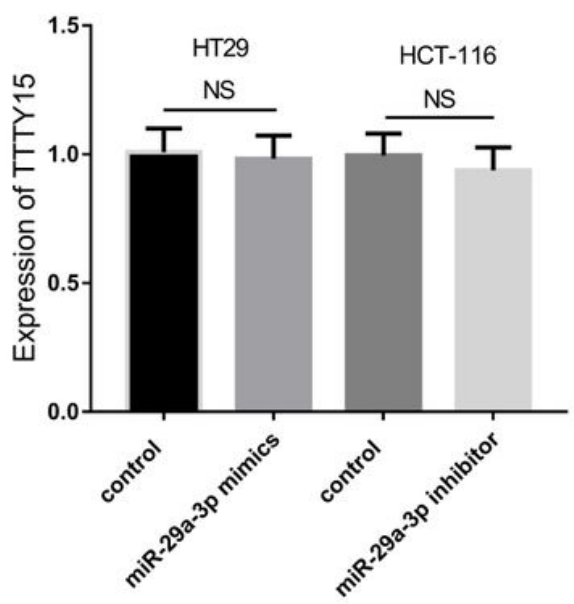

Figure 6

TTTY15 can directly bind to miR-29a-3p to promote the progression of CRC A. It was found that there was two potential binding site between TTTY15 and miR-29a-3p by querying LncBase Predicted V.2 database. B. The binding relationship among TTTY15-wt, TTTY15-mut and miR-29a-3p mimics were verified by luciferase gene reporter assay. C. The expression of TTTY15 and miR-29a-3p in 51 groups of CRC tissues was detected by qRT-PCR, and the results showed that their expression was negatively correlated. D. qRTPCR assay was employed to detect the effect of overexpression and knockdown of TTTY15 on miR-29a$3 p$ expression. E. The effect of overexpression and knock-down of miR-29a-3p on the expression of TTTY15 was detected by qRT-PCR assay. (Compared with control group/NC + vector/NC + shRNA, * indicates $p<0.05$, $* \star$ indicates $p<0.01$, $\star \star \star$ indicates $p<0.001$, and NS indicates $p>0.05$ ) 
A

DVL3-wt :5’ ggACCAGACUU---GUUGGUGCUa 3' II || || | |||||||

miR-29a- 3p :3’ auUGG-CUAAAGUCUACCACGAu 5’

DVL3-mut :5’ ggAGUAUUCAC--AUGACAUCGa 3’
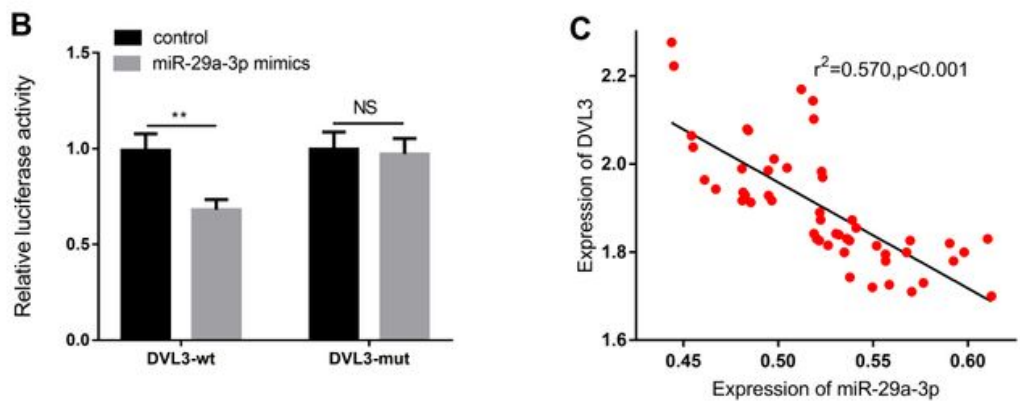

E
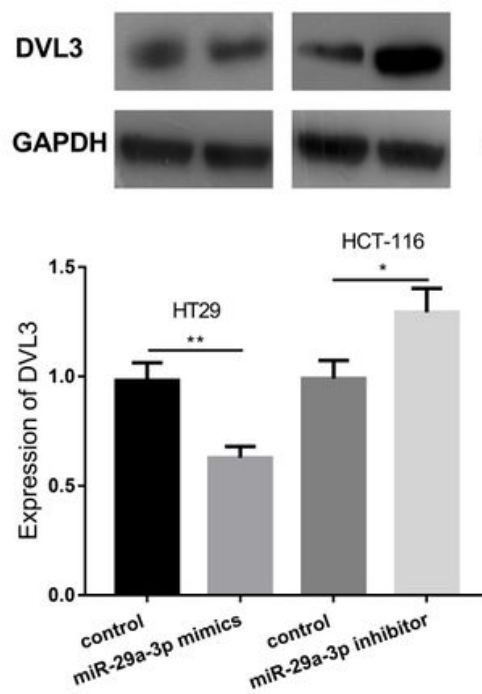

$\mathbf{F}$
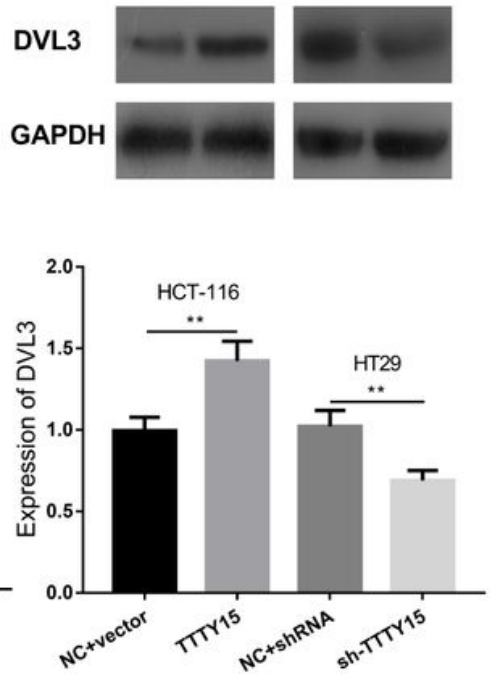

\section{Figure 8}

miR-29a-3p targeted and downregulated DVL3 in CRC A. By querying the Starbase database, it was found that there was a potential binding site between miR-29a-3p and DVL3. B. The binding relationship between miR-29a-3P and DVL3 was verified by luciferase gene reporter assay. C\&D. The expressions of TTTY15, miR-29a-3p and DVL3 in 51 cases of CRC tissues were detected by qRT-PCR, and the correlation between the expression of miR-29a-3p and DVL3, TTTY15 and DVL3 were analyzed. E-F. The effects of miR-29a-3p and TTTY15 expression on DVL3 expression were detected by qRT-PCR and western blot. (Compared with control group/NC + vector/NC + shRNA, * indicates $p<0.05$, ** indicates $p<0.01$, *** indicates $p<0.001$, and NS indicates $p>0.05$ ) 
A

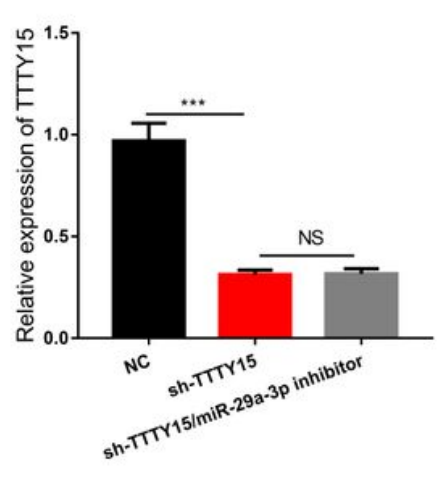

E
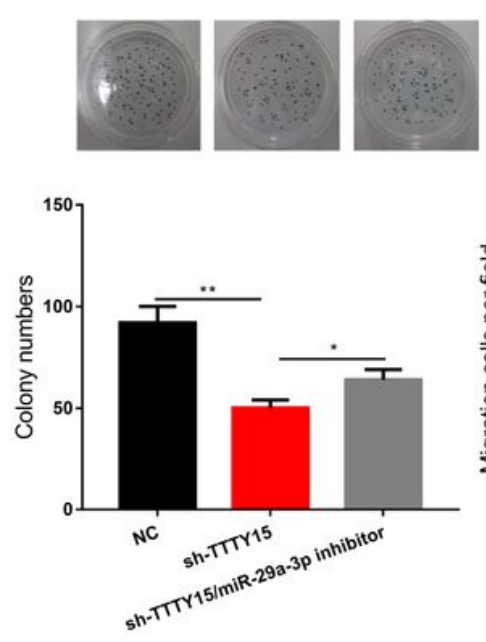

B

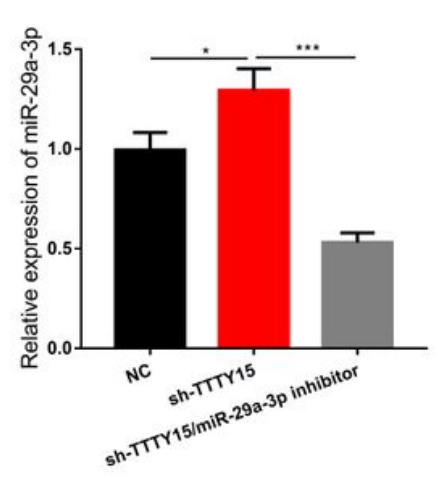

$\mathbf{F}$
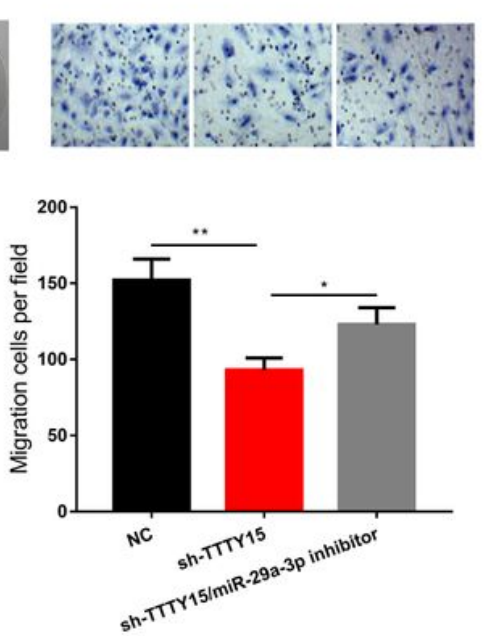

C
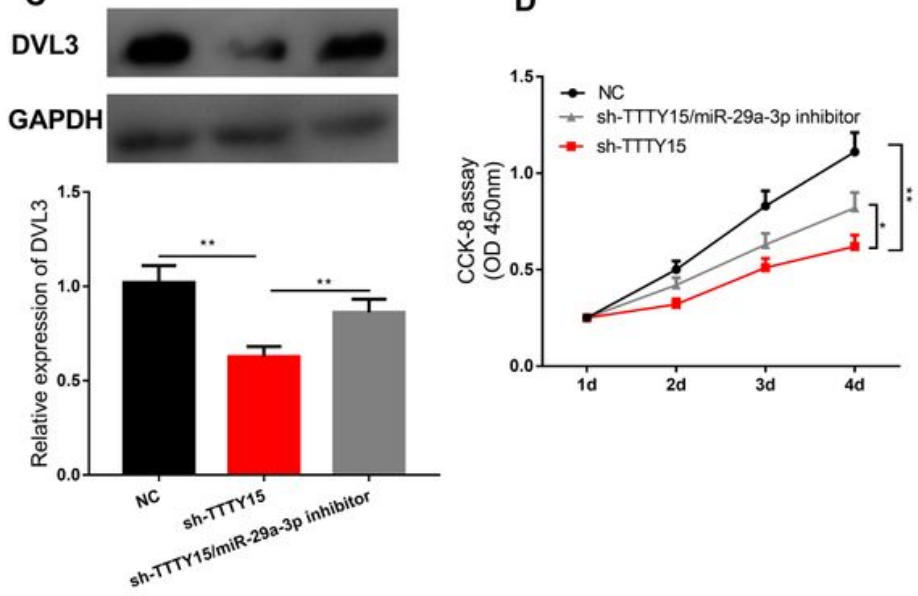

G

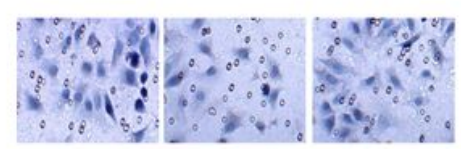

H
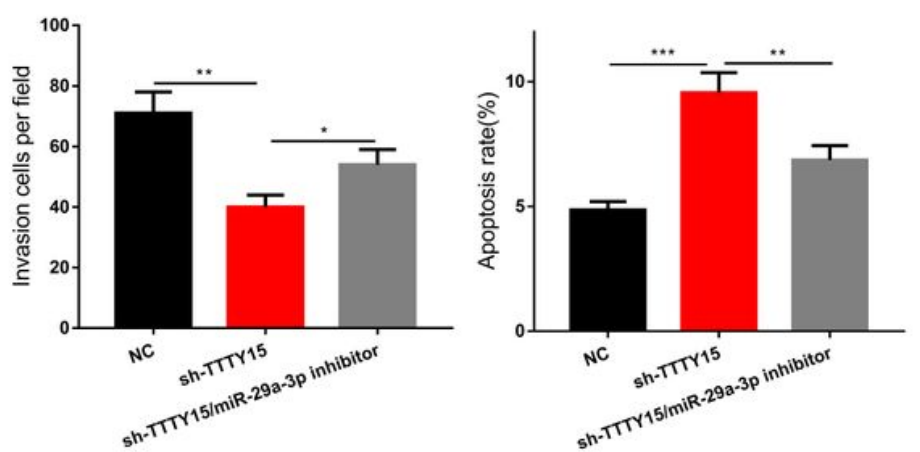

Figure 9

TTTY15 knockdown repressed CRC cell viability, migration, and invasion but induced cell apoptosis via regulating miR-29a-3p A\&B\&C. The expressions of TTTY15, miR-29a-3p and DVL3 in sh-TTTY15 cells and sh-TTTY15/miR-29a-3p inhibitor cells constructed in HT29 cells were detected by qRT-PCR (and western blot). D\&E. CCK-8 method and plate colony formation assay were adopted to detect the proliferation of each group of cells. F\&G. The migration and invasion of cells in each group were detected by Transwell assay. H. Flow cytometry was used to detect the apoptosis in each group. (Compared with $N C$, * indicates $p<0.05, * *$ indicates $p<0.01, * \star \star$ indicates $p<0.001$ ) 